\title{
Hematoma subgaleal crónico en un lactante. Presentación de un caso
}

\author{
J.M. Santín-Amo; M. Gelabert-González; J.M. Villa-Fernández; D. Castro-Bouzas; R. Serramito-García y
} A. García-Allut

Servicio de Neurocirugía Hospital Clínico Universitario de Santiago de Compostela. Departamento de Cirugía. Universidad de Santiago de Compostela.

\section{Resumen}

Los hematomas subgaleales neonatales son colecciones sanguinolentas, localizadas entre la galea y el tejido conectivo epicraneal; con frecuencia son infradiagnosticados, y en la mayoría de las ocasiones guardan relación con determinados procedimientos obstétricos como el uso de fórceps o ventosa. En general tienen poco volumen y suelen solucionarse espontáneamente. Ocasionalmente pueden alcanzar gran tamaño y ponen en riesgo la vida del recién nacido; excepcionalmente tienden a la cronificación siendo necesario para su tratamiento emplear procedimientos quirúrgicos.

Exponemos el caso de una paciente menor de un año de edad que presenta un hematoma subgaleal secundario a parto asistido con ventosa y que precisó tratamiento quirúrgico.

PALABRAS CLAVE. Cefalohematoma. Extracción con ventosa. Galea. Hematoma subgaleal.

Chronic subgaleal hematoma in a child. Case report

\section{Summary}

Neonatal subgaleal hematomas are under-diagnosed collections of blood beneath the galea, often caused by certain obstetric procedures such as use of forceps or vacuum. They generally have low volume and often resolve spontaneously. Occasionally, they can achieve a large volume and may endanger the live of the affected newborns. Rarely, they become chronic and exceptionally they may require surgical treatment.

We report the case of a child under one year of age who was referred to our department because of a subgaleal hematoma secondary to vacuum-assisted delivery that required surgical treatment.

KEY WORDS. Cephalohematoma. Galea. Subgaleal

Recibido: 16-11-10. Aceptado : 21-12-10 hematomas. Vacuum extraction.

\section{Introducción}

Los hematomas subgaleales neonatales son patologías poco frecuentes e infradiagnosticadas en nuestro medio que surgen por sangrado entre la galea y el laxo tejido conectivo epicraneal ${ }^{1}$. En neonatos su origen está en relación con partos distócicos en los que se precisa el empleo de fórceps o ventosa, mientras que en el resto de pacientes pediátricos podrían explicarse por traumatismos mayores o menores, asociados a alteraciones de la coagulación ${ }^{12}$. A diferencia del cefalohematoma, limitado a un único hueso craneal, los hematomas subgaleales pueden cruzar suturas y llegar a extenderse a la totalidad de cráneo, lo que explica en ocasiones su gravedad y una mortalidad en torno al 20-25\% debido a exanguinación, shock hipovolémico e incluso compresión cerebral ${ }^{1,2}$.

\section{Caso clínico}

Paciente nacida a las 39 semanas de edad gestacional, mediante parto instrumentalizado con ventosa, cuyo peso fue $3.980 \mathrm{~g}$. Desde el nacimiento presenta tumoración en línea media del cráneo, que se extiende desde la sutura coronal hasta la región parieto-occipital, de consistencia blanda, compatible con cefalohematoma. Fue tratada de forma conservadora, pero a los 7 meses de edad y dada su persistencia, es remitida a nuestro centro.

En la exploración clínica se observaba un bultoma en línea media craneal de unos $10 \times 7 \times 3 \mathrm{~cm}$, de consistencia blanda, no dolorosa al tacto. El perímetro craneal era de 40 cm. (Figura 1).

Se realizó una radiología simple de cráneo que mostró una colección epicraneal sin afectación subyacente del hueso.

Un estudio con tomografía computarizada (TC) craneal demostró la presencia de una colección hipodensa subcutánea epicraneal, hallazgo que se confirmó mediante una resonancia magnética $(\mathrm{RM})$ que evidenció una colección líquida extracraneal localizada en la línea media, que presentaba morfología semilunar y que ocupaba todo el espa- 


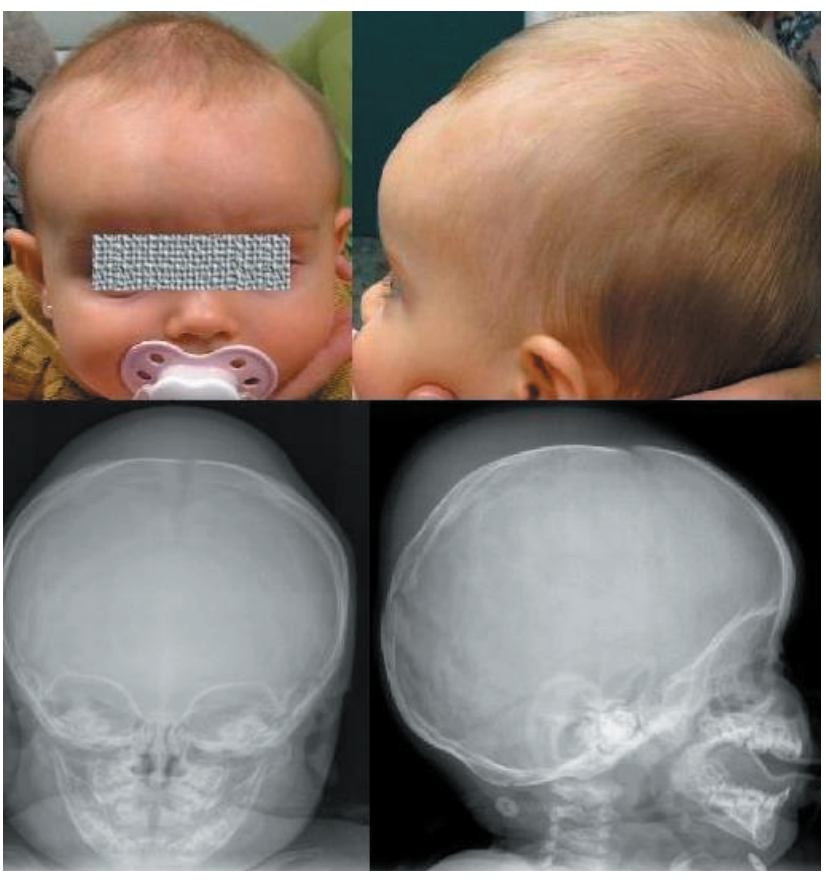

Figura 1. Fotografía de la niña mostrando el bultoma epicraneal. La radiología simple de cráneo reveló una tumoración de tejidos blandos a nivel extracraneal y la ausencia de anomalías óseas.

cio entre las fontanelas anterior y posterior. La lesión se comportaba como hiposeñal en secuencias T1 e hiperseñal en secuencias T2 (Figura 2).

A los 11 meses de edad fue intervenida quirúrgicamente, practicándose una incisión de piel bicoronal, localizándose por debajo de la galea una masa de consistencia blanda rodeada por una cápsula gruesa en la que se incluyó el periostio. Se abrió la capsula dando salida a un contenido de sangre vieja y se procedió a la extirpación completa de la misma.

A los 6 meses de la intervención la niña se encontraba asintomática y no se palpaban masas sobre la calota craneal, considerándose como buen resultado estético.

\section{Discusión}

Durante el parto se pueden producir diversos tipos de lesiones sobre el sistema nervioso central, algunas de las cuales se pueden prevenir ya que están relacionadas con una mala gestión del mismo o al empleo inadecuado del material obstétrico ${ }^{13}$.

El cuero cabelludo esta formado, de fuera a dentro, por cinco membranas principales: piel, capa densa de tejido conectivo, galea, capa laxa de tejido conectivo y periostio; y entre estas membranas pueden originarse diferentes tipos de hemorragias ${ }^{3}$. El caput succedaneum es una colección sero-sanguinolenta que surge entre la piel y la galea, por el

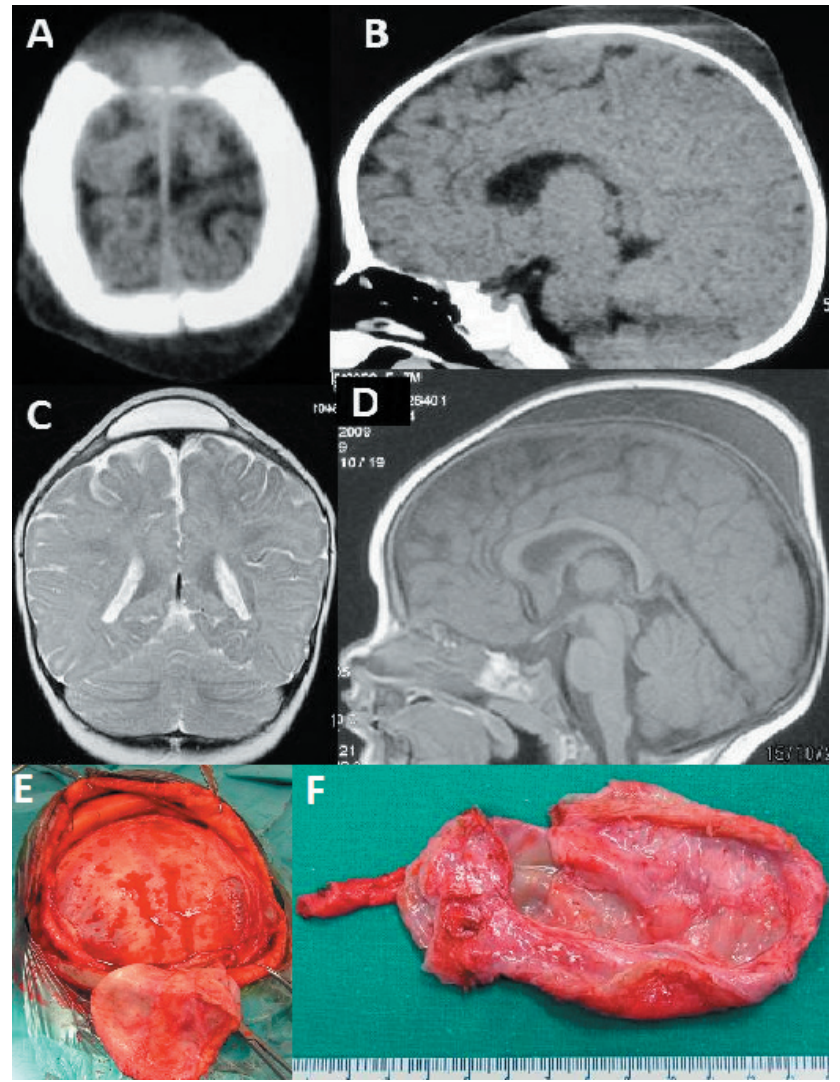

Figura 2. (AB): TC, (CD) RM secuencias T2 y T1 mostrando el bultoma epicraneal. (E) imagen intraoperatoria la que se observa la localización subgaleal de la capsula. (F) aspecto de la capsula extirpada.

contrario el cefalohematoma se desarrolla bajo el periostio y está en relación con la ruptura de venas diploicas ${ }^{5}$.

El hematoma subgaleal ocurre en un espacio rico en venas emisarias, situado entre la galea y el periostio, que conectan los senos intradurales con venas superficiales de la piel. El principal mecanismo patogénico deriva de fuerzas radiales o tangenciales que ocasionan la ruptura de las venas $^{5}$. Su incidencia varía entre 4 a 60 por cada 10.000 nacimientos, aunque también existen casos descritos en pacientes jóvenes e incluso en adultos ${ }^{2,11}$.

En neonatos su origen está en relación con partos complicados en los que se precisa el uso de fórceps o ventosa ${ }^{13}$. La revisión de Chadwick et al de $1996^{4}$ implica al empleo de la ventosa como responsable del 89\% de todos los casos de hematoma subgaleal, mientras que en el resto de pacientes pediátricos estos hematomas podrían explicarse por traumatismos menores, incluido el maltrato infantil ${ }^{9}$. En algunas ocasiones están asociados a alteraciones de la coagulación ${ }^{1}$, a la ingesta de fármacos anticoagulantes o a la de antiagregantes ${ }^{5,7}$. Otros factores de riesgo son la prematuridad, macrosomía, la hipoxia intraútero, la primiparidad y la etnia africana ${ }^{5}$. 
La presencia de este tipo de hematoma en niños de más edad o en adultos ${ }^{10,11}$ está en relación con traumatismos importantes, cirugía craneal previa ${ }^{10}$, ingesta de fármacos antiagregantes/antiocoagulantes ${ }^{6}$ o pueden estar producidos por manipulaciones/tracciones capilares ${ }^{2}$.

Clínicamente, en estadios iniciales, los hematoma subgaleales originan hinchazón difusa de la piel que con el tiempo se transforma en una colección a tensión. La degradación de la hemoglobina retenida en los tejidos puede producir hiperbilirrubinemia e ictericia, sobre todo en niños de menor edad. Dada su capacidad para cruzar suturas pueden, a diferencia del cefalohematoma, extenderse a más de un hueso craneal, lo que explica su facilidad para alcanzar volúmenes que podrían originar situaciones de hipovolemia, shock e incluso compresión cerebral en el neonato ${ }^{2,3}$. Se estima que las pérdidas sanguíneas oscilan en un rango entre $40 \mathrm{ml}$ por cada $\mathrm{cm}$ que aumenta la circunferencia craneal a $260 \mathrm{ml}$ por $\mathrm{cm}$ de aumento del grosor de la piel ${ }^{8}$.

En la mayoría de los casos, sobre todo en recién nacidos, basta la exploración clínica para establecer el diagnóstico, pero en situaciones diferentes, como son pacientes de más edad, o como en nuestro caso donde no se produjo una resolución espontánea, es necesario recurrir a la realización de pruebas de neuroimagen como un TAC y/o RM craneal para establecer el diagnóstico preciso. El nivel de hemoglobina puede no reflejar la gravedad de la hemorragia puesto que, en ocasiones, el sangrado se desarrolla tan rápidamente que el tiempo es insuficiente para producir hemodilución ${ }^{2}$.

Normalmente la actitud suele ser expectante ya que el pronóstico es favorable y el hematoma suele resolverse, sin complicaciones, de forma espontánea en 3-4 semanas.

Sin embargo, en alguna ocasión, el hematoma no es autolimitado y tiende a la cronificación, con la formación de una membrana que lo aísla del resto de las estructuras (cráneo y tejido subcutáneo), lo que obliga a una actitud más agresiva.

Las opciones de tratamiento incluyen la punción y aspiración seguidas de un vendaje compresivo. Existe controversia sobre la indicación de las punciones evacuadoras, en general no se consideran necesarias excepto en el caso de existir importante compresión sobre el cráneo o ante la presencia de datos de hipertensión intracraneal ${ }^{2,9}$.

La necesidad de realizar un abordaje quirúrgico directo, como en nuestro caso, es excepcional ${ }^{7}$. En nuestra paciente consideramos que precisaba la realización de un colgajo cutáneo que permitiera, no solamente la evacuación del contenido hemático, sino también la extirpación de la cápsula que se había formado y la remodelación del cuero cabelludo para obtener un buen resultado cosmético.

\section{Bibliografía}

1. Alcover, E., Jordán, I., Quintillá, J.M., Rodríguez,
J.M., Figueras, J.: Hematoma subgaleal en un recién nacido afecto de hemofilia grave. An Esp Pediatr 1995; 51: 287 289.

2. Amar, A.P., Aryan, H.E., Meltzer, H.S., Levy, M.L.: Neonatal subgaleal hematoma causing brain compression: report of two cases and review of the literature. Neurosurgery 2003; 52: 1470-1474.

3. Boumahn, B., Ghazouni, J., Bey, K.J., Carbonnier, M., Staquet, S.: Hématome du scalp chez 2 nouveau-nés. Arch Ped 2010; 17: 1451-1454.

4. Chadwick, L.M., Pembeton, P.J., Kurinczuk, J.J.: Neonatal subgaleal haematoma: associated risk factors, complications and outcomes. J Pediatr Child Health 1996; 32: 228232.

5. Fujisawa, H., Yonaha, H., Oka, Y, et al.: A marked exophtalmos and corneal ulceration caused by delayed massive expansion of a subgaleal hematoma. Childs Nerv System 2005; 21: 489-492.

6. Greco, F., Fiumara, A., Sorge, G., Pavone, L.: Subgaleal hematoma in a child with Sturge-Weber syndrome: to prevent stroke-like episodes, is treatment with aspirin advisable? Childs Nerv Syst 2008; 24: 1479-1481.

7. Ryan, T.D., Dimmitt, R.A., Tubbs, R.S., et al.: Contribution of surgical evacuation of a large subgaleal hematoma to the resolution of severe conjugated hyperbilirrubinemia in a neonate. J Neurosurg 2007; 106: 131-133.

8. Robinson, R.J., Rossiter, M.A.: Massive subaponeurotic hemorrhage in babies of African origin. Arch Dis Child 1968; 43: 684-687.

9. Seifert, D., Püschel, K.: Subgaleal hematoma in child abuse. Forensic Science International 2006; 131-133.

10. Shehu, B.B.: Massive chronic subgaleal haematoma. Br J Neurosurg 2002; 16: 396-397.

11. Sudhakar, N., Simpson, B.A.: Massive subgaleal haematoma. Br J Neurosurg 2002; 16: 394-395.

12. Strauss T, Kenet G, Schushan-Eisen I ,Mazkereth R, Kuint J: Rescue Recombinant Activated Factor VI for neonatal subgaleal hemorrhage. IMAJ 2009; 11: 639-640.

13. Villarejo, F., Belinchón, J.M., Carceller, F., GómezSierra, A., Pascual, A., Cordobés, F., Pérez-Días, C., Rivero, B.: Lesiones craneales secundarias a parto asistido con fórceps. Neurocirugía 2009; 20: 262-264.

Santín-Amo, J.M.; Gelabert-González, M.; Villa-Fernández, J.M.; Castro-Bouzas, D.; Serramito-García, R.; García-Allut, A.: Hematoma subgaleal crónico en un lactante. Presentación de un caso. Neurocirugía 2011; 22: 261-263.

Correspondencia: Dr. José María Santín Amo. Servicio de Neurocrugía. Complejo Hospitalario Universitario de Santiago. A Choupana s/n. 15706 Santiago de Compostela 